\title{
CdSe/ZnS Colloidal Quantum Dots with Alloyed Core/Shell Interfaces: A Photoluminescence Dynamics Study
}

\author{
K. Dziatkowski ${ }^{a, b, *}$, D. Ratchford ${ }^{a}$, T. Hartsfield ${ }^{a}, \mathrm{X} \cdot \mathrm{Li}^{a}, \mathrm{Y} . \mathrm{GaO}^{c}$ And Z. TAnG $^{c}$ \\ ${ }^{a}$ Department of Physics, University of Texas at Austin, Austin TX 78712, USA \\ ${ }^{b}$ Faculty of Physics, University of Warsaw, Hoża 69, 00-681 Warsaw, Poland \\ ${ }^{c}$ National Center for Nanoscience and Technology, 100190 Beijing, P.R. China
}

\begin{abstract}
Time-resolved photon counting technique was employed to study dynamics of photoluminescence from the ensemble and single $\mathrm{CdSe} / \mathrm{ZnS}$ quantum dots with the alloyed core/shell interfaces. The ensemble data revealed enhanced effect of disorder-induced trap states for increasing emission energy, as implied from the changes in the distribution of total decay rates. The emission trajectories collected for single quantum dots showed familiar, two-state blinking pattern. It suggests that in a large-band-offset CdSe/ZnS system, the introduced alloying of the core-to-shell region cannot smooth enough the confinement potential in order to suppress nonradiative Auger recombination and blinking.
\end{abstract}

PACS: 78.67.Hc, 78.47.jd, 78.55.Et

\section{Introduction}

The colloidal quantum dots (QDs) have been a subject to extensive studies driven by their promising photonic or photovoltaic applications. Nevertheless, their optical properties are not yet optimal from the viewpoint of technological utilization. One of the main unresolved issues is fluctuations of photoluminescence (PL), or so-called "blinking". Despite intense experimental and theoretical research effort, the detailed microscopic mechanism of PL intermittency remains obscure, and so far no single theoretical model accounts for all observations [1]. The most comprehensive explanation postulates that when a QD is charged, a non-radiative Auger ionization suppresses PL and makes the emitter appearing dark [2]. Recently, the diminishing of the Auger process and, in consequence, quenching of blinking was observed in $(\mathrm{Cd}, \mathrm{Zn}) \mathrm{Se} / \mathrm{ZnSe}$ QDs, even though these QDs possessed extra charge [3]. Subsequent theoretical study claimed this observation to be a general feature of the emitters with smoothed quantum confinement potential, since under such conditions the transition matrix element for the Auger recombination is greatly reduced [4]. This paper reports a comprehensive study of PL dynamics in chemically-synthesized CdSe/ZnS QDs with alloyed core/shell interfaces. Due to the softening of the structural interface between the core and the shell such emitters manifest a quantum yield of up to $80 \%$ [5]. The employed technique of time-correlated single photon counting enabled measurements of the decay of exciton luminescence from both the ensemble and individual QDs.

* corresponding author; e-mail: konrad.dziatkowski@fuw.edu.pl

\section{Experimental details}

The QDs studied in this paper were chemically synthesized with a method following Refs. [5, 6] with only marginal modifications. In $20 \mathrm{~mL}$ of 1-octadecene a $0.2 \mathrm{mmol}$ of cadmium oxide, $2 \mathrm{mmol}$ of zinc acetate and $5.5 \mathrm{ml}$ of oleic acid $(\mathrm{OA})$ were dispersed. At $30{ }^{\circ} \mathrm{C}$ the mixture yielded a solution of $\mathrm{Cd}(\mathrm{OA})_{2}$ and $\mathrm{Zn}(\mathrm{OA})_{2}$. Then $0.1 \mathrm{mmol}$ of selenium and $1.5 \mathrm{mmol}$ of sulfur (dissolved in $1.5 \mathrm{~mL}$ of trioctylphosphine) were added and heating continued at $310^{\circ} \mathrm{C}$ for $10 \mathrm{~min}$. After the crystallization process was interrupted by cooling down the solution to $60^{\circ} \mathrm{C}$, the QDs were purified with hexane and acetone. As determined from the atomic force microscopy data the average QD diameter was $9.6 \pm 1.4 \mathrm{~nm}$. Described method was shown to produce CdSe/ZnS QDs with compositional gradation of core-to-shell transition, as inferred from combined high resolution-transmission electron microscopy and inductive coupled plasma-atomic emission spectroscopy studies [5]. Consequently, one expects that QD's confinement potential will also change radially which was schematically depicted in Fig. 1a.

For the purpose of PL dynamics measurements, QDs were excited with $440 \mathrm{~nm}$ Ti:sapphire laser, whose light was focused on the sample (down to the diffraction limit) by $60 \times$ microscope objective. The emission was collected by the same objective and fed into the spectrograph equipped with both charge-coupled device (CCD) camera, for taking spectra, and avalanche photodiode (APD), for time-correlated single photon counting. The spectroscopy of ensemble was performed with $2.5 \mathrm{~nm}$ $(\approx 10 \mathrm{meV})$ resolution thanks to limiting the width of spectrograph's input slit to $200 \mu \mathrm{m}$. After taking a spec- 
(a)
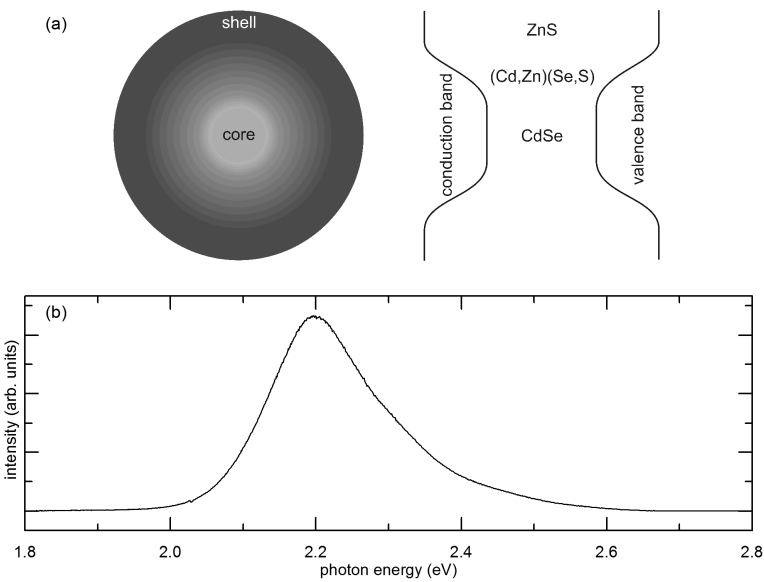

Fig. 1. (a) Schematic of a QD with an alloyed core/ shell interface. (b) PL spectrum of the ensemble of QDs under study.

trum with CCD camera, Fig. 1b, the emission decay rate was measured versus the emitted photon energy by rotating the spectrograph's grating and selecting different portions of the spectrum that were directed to APD. Such spectral selection enabled measurements on QDs with similar emission energy (thus with similar diameter) within the ensemble. The PL dynamics measurements on single QDs were performed in time-tagged time resolved mode that allowed for simultaneous acquiring long-time-scale blinking trajectories with $10 \mathrm{~ms}$ resolution and short-time-scale PL decays with 500 ps resolution. Single dots were accessed by spin coating the colloid with a surface density of about $0.01 \mu \mathrm{m}^{-2}$ and using the laser spot of less than $1 \mu \mathrm{m}$ diameter. Then by means of the atomic force microscopy only those luminescent objects were selected that revealed two-level blinking trajectories (as opposed to the multi-level trajectories of QDs aggregates).

\section{Results and discussion}

In Fig. 2a the measured decay curve of ensemble PL is presented together with two theoretical curves fitted according to different models. The easily noticeable discrepancy from single-exponential dependence was found to be a qualitatively general feature of QDs with any emission energy from the batch in study. In order to give a better than single-exponential quantitative description of total decay rates, a phenomenological model was applied, where a general decay function is given by [7]:

$$
f(t)=\int_{0}^{\infty} \rho(\Gamma) \mathrm{e}^{-\Gamma t} \mathrm{~d} \Gamma .
$$

Here $\Gamma$ denotes the total decay rate that follows $\log$ normal distribution given by

$$
\begin{aligned}
& \rho(\Gamma)=\frac{1}{\Gamma_{\mathrm{mf}} w \sqrt{\pi} \exp \left(w^{2} / 4\right)} \\
& \times \exp \left(-\left(\frac{\ln \Gamma-\ln \Gamma_{\mathrm{mf}}}{w}\right)^{2}\right),
\end{aligned}
$$

where $\Gamma_{\mathrm{mf}}$ is the most frequent decay rate and $w=$ $\operatorname{asinh}\left(\Delta \Gamma / 2 \Gamma_{\mathrm{mf}}\right)$ can be expressed by the width $\Delta \Gamma$ of $\log$-normal distribution at $1 / \mathrm{e}$ of its height. As clearly seen in Fig. 2a the log-normal approach provides better fit than single-exponential decay, which may be expected for measurements done on the ensemble of QDs with different diameters and most probably with different total decay rates. The latter conjecture is confirmed by Fig. 2b, where dependences of $\Gamma_{\mathrm{mf}}$ and $\Delta \Gamma$ on the emission energy are plotted. Both quantities increase with rising photon energy $E$, i.e. with diminishing size of a quantum dot.
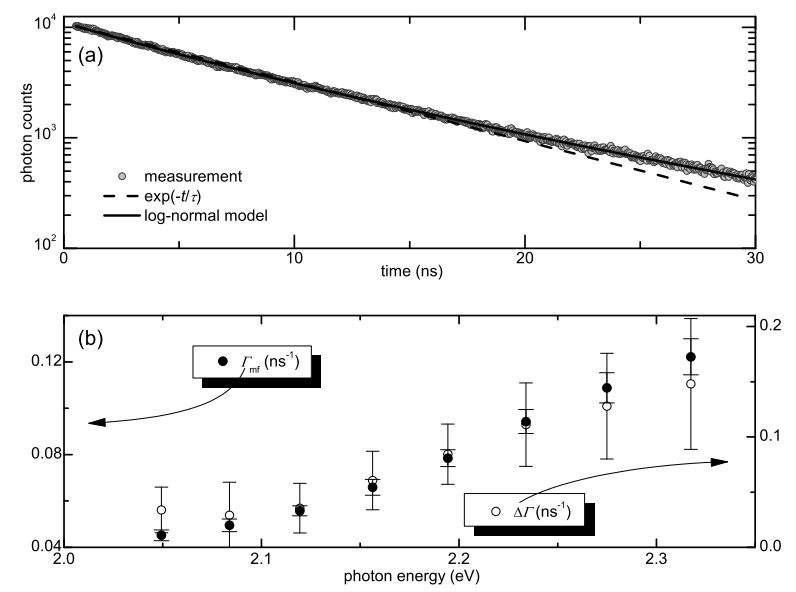

Fig. 2. (a) PL decay of the ensemble with fitted single-exponential ( $\tau=8.1 \mathrm{~ns}$ ) and $\log$-normal distribution $\left(\Gamma_{\mathrm{mf}}=0.11 \mathrm{~ns}^{-1}, \Delta \Gamma=0.13 \mathrm{~ns}^{-1}\right)$ models. (b) Dependence of log-normal distribution parameters on the ensemble emission energy.

Being a measure of the total decay rate $\Gamma_{\mathrm{mf}}$ contains both radiative, $\Gamma_{\text {rad }}$, and nonradiative, $\Gamma_{\text {nrad }}$, contributions, the former of which is known to increase with photon energy [8]. Dependence of the latter component on $E$ is directly related to the microscopic details of dominant nonradiative mechanism. The trap states located at surface/interface are one of the most important contributors to $\Gamma_{\text {nrad }}$, so their influence should increase with growing surface-to-volume ratio, i.e. with decreasing diameter and increasing $E$. Thus $\Gamma_{\text {rad }}, \Gamma_{\text {nrad }}$, and - in consequence - $\Gamma_{\mathrm{mf}}$ may be expected to increase with the emission energy $[4,9,10]$.

The other parameter of log-normal distribution, $\Delta \Gamma$, similarly increases with $E$ and such dependence also can be ascribed to growing surface-to-volume ratio. Each data point in Fig. 2b was obtained with the spectrograph's input slit narrowed enough to limit contribution 
from QDs with different sizes, i.e. with different $\Gamma_{\text {rad }}$. Consequently, the change of $\Delta \Gamma$ with $E$ relates mainly to changes in the distribution of $\Gamma_{\text {nrad }}$. Disorder-induced trap states in the core-to-shell transition are often indicated as the most important factor in $\Gamma_{\text {nrad }}[11,12]$. Their influence on the excitons (or the influence of any compositional fluctuations in the core-to-shell area) should be more efficient for small QDs with large surface-to-volume ratio, i.e. those with higher emission energy, resulting in broader distribution of $\Gamma_{\text {nrad }}$.

In order to identify possible blinking behavior, the $\mathrm{PL}$ dynamics for single QDs selected from the ensemble was studied. In Fig. 3a a typical PL spectrum of a single QD under study is presented, whereas Fig. 3b shows the emission trajectory of a given dot. In the latter figure the intermittency of emission with distinct "on" and "off" states is apparent, despite intentional alloying of the core-to-shell transition*. Recently, the suppression of blinking in ( $\mathrm{Cd}, \mathrm{Zn}) \mathrm{Se} / \mathrm{ZnSe}$ nanocrystals with compositional gradation was reported and interpreted in terms of alloying-induced softening of the confinement potential [3]. For the efficient Auger recombination (and blinking) to arise, the steep potential changes are needed in order to relax momentum conservation rules [13], whereas with the smooth band profile the intermittency of PL will be quenched even for charged emitters [3]. Since CdSe/ ZnS QDs investigated in this study comprise of semiconductors with much larger core/shell band gap offset as compared to those in the work of Wang et al., then the carriers in $\mathrm{CdSe} / \mathrm{ZnS}$ system perceive a stronger confinement within a steeper band profile. Under such conditions the Auger process may persist as the efficient nonradiative recombination path. The above discussion is supported by the recent theoretical findings based on quantitative model of the core/shell ionization energy offset [14], where $\mathrm{CdSe} / \mathrm{ZnS}$ system was identified as the least likely to reveal the alloying-induced suppression of blinking. Similarly, the analysis based on a two-band, effective mass Kane model led to the conclusion that for a given functional form of the core-to-shell potential the Auger recombination becomes more efficient with the increasing confinement steepness [4].

Finally, the radiative decay time was estimated for QDs under study. The PL decay curve - even when measured for a single QD - is often of multi-exponential nature due to blinking-related fluctuating charging events [2]. As seen in Fig. 3c, it becomes single-exponential when the analyzed emission is limited to "on"-states only; see threshold in Fig. 3b [11]. Then on semi-log scale one may obtain a good linear fit with a characteristic decay time of about 35 ns for a given dot and about $29 \pm 3$ ns when averaged over

\footnotetext{
* The non-zero "off"-state signal, whose main contributors are ambient light and APD dark count, is comparable with the background count rate measured when the confocal spot is not focused on any QD.
}
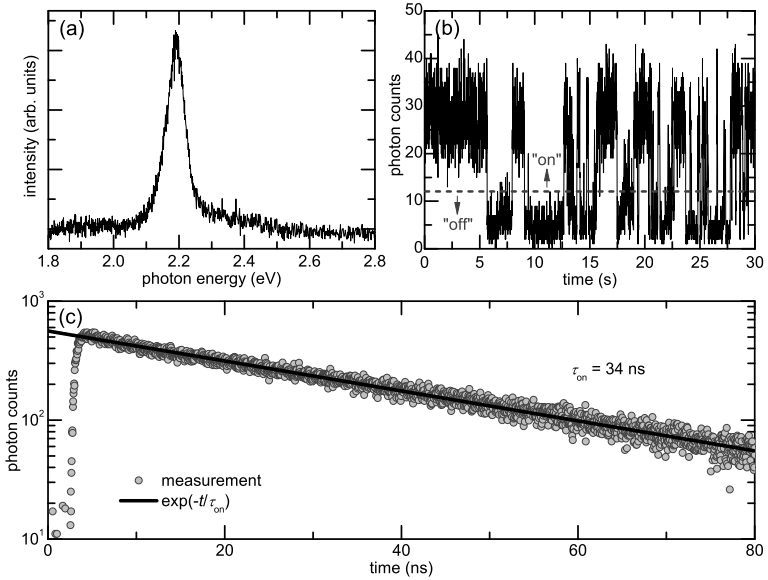

Fig. 3. (a) PL spectrum of a single QD. (b) Trajectory of emission from a single QD. The arbitrarily chosen threshold discriminates "on" and "off" states of the QD. (c) PL decay limited to "on"-states only with fitted single-exponential model.

many single QDs. The latter number corresponds to $\Gamma_{\mathrm{rad}}=0.035 \pm 0.004 \mathrm{~ns}^{-1}$ and - as expected - is smaller than any of $\Gamma_{\mathrm{mf}}$ values depicted in Fig. 2b, since $\Gamma_{\mathrm{mf}}$ (being an estimate of total decay rate) contains contributions from both slow radiative as well as fast nonradiative processes.

\section{Conclusions}

The comprehensive PL dynamics study was performed on CdSe/ZnS QDs with the alloyed core-to-shell interfaces. Time-tagged time resolved single photon counting technique was employed in order to collect both spectroscopic and decay data for the ensemble and single QDs. The ensemble measurements revealed - for increasing emission energy - a systematic rise of the total decay rates with greater variation, which was attributed to the enhanced effect of disorder-induced surface/interface trap states in QDs with smaller diameter. The single QD data showed familiar blinking behavior with distinct "on" and "off" states, despite compositional modification of the core-to-shell transition. This observation was explained in terms of large difference of the energy gaps between CdSe and ZnS. The large band gap offset promotes nonradiative Auger recombination and, in consequence, alleviates diminishing effect of gradation on blinking.

\section{References}

[1] P. Frantsuzov, M. Kuno, B. Janko, R.A. Marcus, Nature Phys. 4, 519 (2008).

[2] A.L. Efros, M. Rosen, Phys. Rev. Lett. 78, 1110 (1997).

[3] X. Wang, X. Ren, K. Kahen, M.A. Hahn, M. Rajeswaran, S. Maccagnano-Zacher, J. Silcox, G.E. Cragg, A.L. Efros, T.D. Krauss, Nature 459, 686 (2009). 
[4] G.E. Cragg, A.L. Efros, Nano Lett. 10, 313 (2010).

[5] W.K. Bae, K. Char, H. Hur, S. Lee, Chem. Mater. 20, 531 (2008).

[6] W.K. Bae, J. Kwak, J.W. Park, K. Char, C. Lee, S. Lee, Adv. Mater. 21, 1690 (2009).

[7] A.F. van Driel, I.S. Nikolaev, P. Vergeer, P. Lodahl, D. Vanmaekelbergh, W.L. Vos, Phys. Rev. B 75, 035329 (2007).

[8] A.L. Efros, Phys. Rev. B 46, 7448 (1992).

[9] M.D. Leistikow, J. Johansen, A.J. Kettelarij, P. Lodahl, W.L. Vos, Phys. Rev. B 79, 045301 (2009).

[10] A.F. van Driel, G. Allan, C. Delerue, P. Lodahl, W.L. Vos, D. Vanmaekelbergh, Phys. Rev. Lett. 95, 236804 (2005).
[11] B.R. Fisher, H.J. Eisler, N.E. Stott, M.G. Bawendi, J. Phys. Chem. B 108, 143 (2004).

[12] M.G. Bawendi, P.J. Carroll, W.L. Wilson, L.E. Brus, J. Chem. Phys. 96, 946 (1992).

[13] A.L. Efros, D.J. Lockwood, L. Tsybeskov, Semiconductor Nanocrystals: from Basic Principles to Applications, Kluwer Academic/Plenum Publ., New York 2003.

[14] A.D. Arulsamy, U. Cvelbar, M. Mozetic, K. Ostrikov, Nanoscale 2, 728 (2010). 